\title{
Using textual clues to improve metaphor processing
}

\author{
Stéphane Ferrari \\ LIMSI-CNRS \\ PO Box 133 \\ F-91403 Orsay cédex, France \\ ferrari@limsi.fr
}

\begin{abstract}
In this paper, we propose a textual clue approach to help metaphor detection, in order to improve the semantic processing of this figure. The previous works in the domain studied the semantic regularities only, overlooking an obvious set of regularities. A corpus-based analysis shows the existence of surface regularities related to metaphors. These clues can be characterized by syntactic structures and lexical markers. We present an object oriented model for representing the textual clues that were found. This representation is designed to help the choice of a semantic processing, in terms of possible non-literal meanings. A prototype implementing this model is currently under development, within an incremental approach allowing step-by-step evaluations. ${ }^{1}$
\end{abstract}

\section{Introduction}

Metaphor is a frequently used figure of speech, reflecting common cognitive processes. Most of the previous works in Natural Language Understanding (NLU) looked for regularities only on the semantic side of this figure, as shown in a brief overview in section 2. This resulted in complex semantic processings, not based on any previous robust detection, or requiring large and exhaustive knowledge bases. Our aim is to provide NLU systems with a set of heuristics for choosing the most adequate semantic processing, as well as to give some probabilistic clues for disambiguating the possibly multiple meaning representations.

A corpus-based analysis we made showed the existence of textual clues in relation with the metaphors. These clues, mostly lexical markers combined with syntactic structures, are easy to spot, and can provide a first set of detection heuristics. We propose, in

\footnotetext{
${ }^{1}$ This work takes part in a research project sponsored by the AUPELF-UREF (Francophone Agency For Education and Research)
}

section 3 , an object oriented model for representing these clues and their properties, in order to integrate them in a NLU system. For each class, attributes give information for spoting the clues, and, when possible, the source and the target of the metaphor, using the results of a syntactic parsing. A prototype, STK, partially implementing the model, is currently under development, within an incremental approach. It is already used to evaluate the clues relevance.

In conclusion, we will discuss how the model can help chosing the adequate semantic analysis to process at the sentence level or disambiguating multiple meaning representations, providing probabilities for non-literal meanings.

\section{Classical methods: a brief overview}

The classical NLU points of view of metaphor have pointed out the multiple kinds of relations between what is called the source and the target of the metaphor, but rarely discuss the problem of detecting the figure that bears the metaphor. For our purpose, we choose to present these approaches in two main groups, depending on how they initiate the semantic processing.

The previous works led to a classification introduced by Dan Fass (Fass, 1991). In the comparison view, the metaphor corresponds to an analogy between the structures representing the source and the target of the figure, as in Gentner's works (Gentner, 1988) and their implementation (Falkenhainer et al., 1989). The interaction view, as in Hobbs (Hobbs, 1991), points at the novelty brought by the metaphor. Fass also distinguishes a selection restrictions violations view presenting the metaphor as a kind of anomaly. We would argue that the two previous views already considered metaphor as a kind of anomaly. Indeed, the semantic analysis proposed for dealing with metaphors were processed depending on the results of another, say a "classical" one $^{2}$.

\footnotetext{
${ }^{2}$ We prefer to call it a classical rather than literal meanings processing because it can deal with some conventional metaphors, even if not explicitly mentioned.
} 
Thereby, detecting a metaphor meant detecting an anomaly in the meaning representation issued from such a classical analysis.

Fass proposed a method for discriminating literal meanings, metaphors, metonymies and "anomalies", merging different points of view (Fass, 1991). In this approach, multiple semantic analysis can be processed, resulting in possibly multiple meaning representations. In (Prince and Sabah, 1992), a method to overcome similar kinds of ambiguities reveal the difficulties encountered if no previous detection is made. James Martin's approach (Martin, 1992), called the conventional view by Fass, is based on Lakoff's theory on cognitive metaphors (Lakoff and Johnson, 1980). It requires a specific knowledge representation base and also results in multiple representation meanings. Detecting a metaphor is meaningless here, and conventional metaphoric meanings can be viewed as polysemies. Martin revealed at least that the heuristic of the ill-formness of meaning representations issued from classical analysis is not sufficient at all to deal with all the possible metaphors.

In our point of view, all the previous approaches were founded. The main remaining problem, however, is to choose an adequate processing when confronted with a metaphor, and thus, to detect the metaphors before trying to build their meaning representation. This can be partially solved using textual clues.

\section{Textual clues: object oriented description}

If the classical views of the metaphor overlook the textual clues, in other domains, especially those concerning explanation, they have been wisely reintroduced. In (Pery-Woodley, 1990), Pery-Woodley shows the existence of such clues related to the explanatory discourse. They can help in generating explanations in natural language as well as in modelling the student in a intelligent tutoring system (Daniel et al., 1992). A corpus of 26 explanatory texts in French, of about 200 words each, has been collected under a shared research project between psychologists and computer scientists, in order to study metaphors and analogies in teaching. The analysis we made showed the existence of textual clues in relation with metaphoric contexts and analogies (e.g. "like", "such as", "illustrated by"). They can be characterized by syntactic regularities (e.g. the comparative is used in structures such as "less than", "more than"; the identification is made through attributes or appositions, ...). They also involve lexical markers (e.g. "literaly", "illustrating", "metaphorically",). These properties, already found in the previous works, can help detecting the clues themselves. Studying the relation between the syntactic regularities and the lexical markers, one can observe that the first build the ground where to find the second. We thus propose an object-oriented model for representing these clues. A generic textual clue can thereby be described by the two following attributes:

- the Surface Syntactic Pattern representing the syntactic regularity, with a label on the item where to find the lexical marker

\section{- the Lexical Marker itself}

Typically, the word "metaphor" itself can be used as a lexical marker in expressions such as "to extend the conventional metaphor, pruning such a tree means to generalize". On the other hand, "metaphor" will not be a marker if used as the subject of the sentence, like in this one. Thus, describing the syntactic regularities surrounding a lexical marker improves its relevance as a marker. We propose to represent this relevance for probabilistic purposes. Each clue that was found is currently evaluated on a large corpus (about 450,000 words). The frequencies of use of the lexical markers in metaphoric contexts are represented in the relevance attribute (see example below).

The syntactic structures may also give information about the source and the target of the metaphor. For instance, in the sentence "Yesterday, at home, Peter threw himself on the dessert like a lion.", the subject inherits the properties of speed and voracity of a lion attacking its victim. It is here possible to spot the source and the target of the metaphor using the syntactic properties of the comparison. Two attributes are added to textual clues related to metaphors, corresponding to the elements of the sentence bearing the source and the target.

\section{Example of textual clue representations}

type metaphor-analogy

name $B .2 .2 .2$

comment comparison involving the meaning of a marker, adjective, attribute of the object, object before the verb

$\begin{array}{lllllll}\text { SSP } & G N_{0} & G N_{1} & V_{1} & A d j_{0} & {[p r e p}\end{array} \quad G N_{2}$

LM $A d j_{0}$ : pareil (meaning "similar")

target $G N_{1}$

source $G N_{2}$

LM relevance $(15 / 28)$

number of occurrences 28

conventional metaphors 3

new metaphors 2

metaphoric contexts 12

total 15

Notations: $G N$ and $G V$ stand for nominal or verbal groups, $A d j$ and $A d v$ for adjectives and adverbs, and prep for prepositions.

The model has been partially implemented in a tool, STK, for detecting the textual clues related to 
metaphors and adding specific marks when found. In its current version, STK allows us to tokenize, tag, and search for lexical markers on large corpora. The tagger we use is the one developped by Eric Brill (Brill, 1992) with a set of tags indicating the grammatical categories as well as other information such as the number and the gender for nouns and adjectives. It is evaluated under $\mathrm{GRACE}^{3}$ protocol for corpus-oriented tools assigning grammatical categories. It is currently used for the evaluation of the textual clues that were found. The latter can be easily retrieved using STK, avoiding lexical ambiguities. They are then analyzed by hand, in order to determine their relevance attribute. In the previous example of textual clue, the relevance values are issued from this corpus-based analysis.

\section{Conclusion, perspectives}

Classical approaches to the metaphor in NLU revealed multiple underlying processes. We therefore focussed our study on how to help detecting metaphors in order to chose the most adequate semantic processing. Textual clues can give information about the figures that bear the metaphor, which are easy to spot. Indeed, they can be found using the results of syntactic parsing. We proposed an object-oriented model to represent these clues and their multiple properties.

If textual clues give information about possible non-literal meanings, metaphors and analogies, one may argue they do not allow for a robust detection. Indeed, a textual clue is not sufficient to prove the presence of such figures of speech. The relevance of each clue can be used to help disambiguating multiple meaning representation when it occurs. This must not be the only disambiguation tool, but when no other is avalaible, it provides NLU systems with a probabilistic method.

Our future works will focuss on the study of the relation between the metaphors introduced by a clue and others that are not conventional. The guideline is that novel metaphors not introduced by a clue at the sentence level may have been introduced previously in the text.

\section{References}

Brill, E. (1992). A simple rule-based part of speech tagger. In Proceedings of the Third Conference on Applied Natural Language Processing, Trento. ACL.

Daniel, M., Nicaud, L., Prince, V., and PeryWoodley, M. (1992). Apport du style Linguis-

\footnotetext{
${ }^{3}$ GRACE stands for "Grammars and Resources for Corpora Analysis and their Evaluation". It is a national research project for the development of tools for French language processing.
}

tique la Modélisation Cognitive de l'Élève. Lecture Notes in Computer Sciences, 608:252-260. Proceedings of the International Conference on Intelligent Tutoring Systems (ITS-92), Montréal.

Falkenhainer, B., Forbus, K., and Gentner, D. (1989). The Structure-Mapping Engine: Algorithm and Examples. Artificial Intelligence, 41:163.

Fass, D. (1991). met : A Method for Discriminating Metonymy and Metaphor by Computer. Computational Linguistics, 17(1):49-90.

Fass, D., Hinkelman, E., and Martin, J., editors. Proceedings of the IJCAI Workshop on Computational Approaches to Non-Literal Language, Sydney, Australia. 1991.

Gentner, D. (1988). Analogical Inference and Analogical Access, In: Analogica, chapter 3, pages 63-88. Edited by Prieditis A., Pitman Publishing, London, Morgan Kaufmann Publishers, Inc., Los Altos, California.

Hobbs, J. (1991). Metaphor and abduction. In (Fass et al., ), pages 52-61.

Lakoff, G. and Johnson, M. (1980). Metaphors we live by. University of Chicago Press, Chicago, U.S.A.

Martin, J. (1992). Computer Understanding of Conventional Metaphoric Language. Cognitive Science, $16: 233-270$.

Pery-Woodley, M. (1990). Textual clues for user modeling in an intelligent tutoring system. Master's thesis, University of Manchester, England, Great-Britain.

Prince, V. and Sabah, G. (1992). Coping with Vague and Fuzzy Words: A Multi-Expert Natural Language System which Overcomes Ambiguities. In Acts of PRICAI'92, Seoul, Corea. September, 1992. 